\title{
Accuracy and Precision of an Optoacoustic Prototype in Determining Endotracheal Tube Position in Children
}

\author{
Teresa A Volsko MBA MHHS RRT CMTE FAARC, Yuriy Petrov PhD, \\ Neil L McNinch MSc RN PSTAT, Donald S Prough MD, Clark R Anderson MSc, and \\ Michael T Bigham MD
}

\begin{abstract}
BACKGROUND: Confirmation of endotracheal tube (ETT) tip position and timely identification and correction of malposition is an essential component of care for endotracheally intubated and mechanically ventilated children. We evaluated the ability of a prototype optoacoustic medical device to determine ETT tip position. We hypothesized that the precision of optoacoustic assessment of ETT tip position would be comparable to chest radiography. METHODS: We recruited children aged newborn to $16 \mathrm{y}$ who were admitted to the pediatric ICU requiring tracheal intubation and undergoing a chest radiograph for clinical purposes. After positioning each child on a chest radiograph plate, a sterile optical fiber, temporarily inserted through the ETT, emitted laser pulses perpendicular to the fiber and to the ETT, generating acoustic (ultrasound) waves in overlying tissue when the tip of the fiber passed beneath an acoustic sensor in the sternal notch. The distance from the ETT tip to the peak acoustic signal was used to calculate the distance from the ETT tip to the carina, which was compared with the same distance calculated by the radiologist reading the chest radiograph. Pearson's correlation coefficient, paired $t$ tests, a Bland-Altman plot were used to compare the measures $(P<.05$ was considered statistically significant). RESULTS: Twenty-six subjects were enrolled: 15 (57.7\%) were male, median (interquartile range) age, weight, and height were 9 months (4-24), $9.6 \mathrm{~kg}(5.7-13.0)$, and $75 \mathrm{~cm}$ (62-90), respectively. All ETTs were cuffed (internal diameter range 3.0-5.0 mm). The relationship between optoacoustic and chest radiograph measurements was strong $(\mathrm{r}=0.91, P<.001)$. Bias was $0.1 \mathrm{~cm}$ with narrow limits of agreement between measures $(0.58 \mathrm{~cm}$ and $0.76 \mathrm{~cm})$. CONCLUSIONS: The optoacoustic prototype accurately determined ETT tip position and was comparable in precision to chest radiograph. Key words: tracheal intubation; tracheal tube malposition; pediatrics; optoacoustic; chest radiograph. [Respir Care 2018;63(12):1463-1470. () 2018 Daedalus Enterprises]
\end{abstract}

\section{Introduction}

Confirmation of endotracheal tube (ETT) tip position and timely identification and correction of malposition is

\footnotetext{
Ms Volsko is affiliated with the Nursing Administration, and the Rebecca C. Considine Research Institute, Akron Children's Hospital, Akron, Ohio. Dr Petrov is affiliated with the Center on Bioengineering, University of Texas Medical Branch at Galveston, Galveston, Texas. Mr McNinchis affiliatd with the Rebecca D Considine Research Institute, Akron Children's Hospital, Akron, Ohio. Dr Prough is affiliated with the Department of Anesthesiology, University of Texas Medical Branch at Galveston, Galveston, Texas. Mr Anderson is affiliated with the Department of Biostatistics, University of Texas Medical Branch at Galveston, Galveston, Texas. Dr Bigham is affiliated with the Division of Critical Care Medicine, Akron Children's Hospital, Akron, Ohio.
}

an essential component of care for endotracheally intubated and mechanically ventilated children. During endotracheal intubation, it is not possible to visualize the tip once the tip of the tube passes through the vocal cords. ${ }^{1}$ The tip of a properly positioned ETT should be $2-3 \mathrm{~cm}$

\footnotetext{
Drs Prough and Petrov are inventors of the technology and are founders of Prospiria. Dr Bigham and Ms Volsko disclose a relationship with Prospiria. The other authors have no conflicts to disclose.
}

Correspondence: Teresa A Volsko MBA MHHS RRT CMTE FAARC, Nursing Administration, Akron Children's Hospital, Akron, Ohio 44308. E-mail: tvolsko@akronchildrens.org.

DOI: $10.4187 /$ respcare. 06140 
above the carina and the cuff distal to the vocal cords. The tracheal length is approximately $4-6 \mathrm{~cm}$ in infants and 5-10 cm in larger children, ${ }^{2}$ yielding a narrow range of appropriate mid-tracheal position and increasing the risk for placing the ETT too shallow or too deep during intubation.

See the Related Editorial on Page 1575

Verifying the intratracheal position of an ETT is important during elective or emergent intubation and is essential to ongoing clinical care in the pediatric ICU. ${ }^{3-6}$ The initial assessment of ETT tip position consists of physical examination (ie, auscultation of the epigastrium and breath sounds) and may be facilitated by capnography, chest radiography, and light/sound-transmitting devices. ${ }^{7}$ As the most widely accepted method of confirmation, chest radiograph provides a single snapshot of the ETT position, but this method is associated with delays in ordering, performing, and interpreting the image, and it also exposes infants and children to radiation. Diagnostic radiographs are associated with higher long-term malignancy risk in infants and children because they have greater tissue radio-sensitivity. There is also an inverse exponential relationship between the risk of radiation exposure and age at exposure..$^{8-10}$

In addition to the need to immediately confirm ETT position after intubation, there is a subsequent need for frequent assessment and monitoring to maintain the tube's proper position. During a patient's course of treatment, an ETT may be unintentionally advanced or withdrawn, either partially or completely. Neck flexion or extension, poorly secured ETTs, and changes in patient position during routine care, diagnostic procedures, or intrahospital or interfacility transport can contribute to malposition of a previously well positioned ETT. ${ }^{11,12}$ Malposition may compromise ventilation and is associated with significant morbidity and mortality. ${ }^{13}$

Presently, no commercially available device can quickly confirm initial ETT position and provide rapid or continuous confirmation of ETT position in infants and children after the initial positioning. Proceedings from scientific conferences report optoacoustic (OA) technology as a novel approach to confirm ETT position in adults. ${ }^{14}$ Use of an OA prototype with a small sample of adult cardiac surgical subjects provided continuous monitoring of ETT position. ${ }^{14}$ The purpose of this pilot study was to compare the accuracy and precision of chest radiograph with that of an investigational OA prototype (PoET, Prospiria, Galveston, Texas) in determining ETT placement in children. We hypothesized that OA assessment of ETT depth would be comparable to that performed with chest radiograph.

\section{QUICK LOOK}

\section{Current knowledge}

Verifying the intratracheal position of an endotracheal tube is important during elective or emergent intubation and is essential to ongoing clinical care. Although it only provides a single snapshot, the chest radiograph is the most widely accepted method of confirming endotracheal tube position.

\section{What this paper contributes to our knowledge}

Investigational devices using optoacoustic technology showed promise in accurately determining endotracheal tube position and may reduce or eliminate the need for chest radiography to confirm endotracheal tube position.

\section{Methods}

\section{Setting and Study Design}

This prospective single-institution pilot study was performed in the pediatric ICU of Akron Children's Hospital in Akron, Ohio, and utilized a convenience sample. The protocol was approved by the institutional review board, which concluded that the device constituted a minimal risk and that no investigational device exemption was required. Informed consent was obtained prior to study enrollment and participation. Children newborn to $16 \mathrm{y}$ of age who required endotracheal intubation and mechanical ventilatory support were included. Subjects were eligible only if a physician ordered a chest radiograph for routine clinical purposes. Exclusion criteria included the inability to obtain informed consent from the parents or legal guardian and mechanically ventilated children for whom a chest radiograph was not ordered.

\section{Technology and Pre-Study Procedures}

The OA prototype uses a 1.0-mm flexible optical fiber temporarily placed within an ETT to emit high-frequency laser pulses perpendicular to the fiber tip. Upon encountering tracheal tissue, the laser pulses generate ultrasound waves that are detected by an acoustic detector and converted in real time to a graphical depiction of signal amplitude.

For this study, the optical fibers were customized for each ETT's inner diameter and length. When maximally inserted, the distal tip of the optical fiber only extended to the distal border of the ETT cuff. This detail ensured that initial placement of the optical fiber's distal tip would not extend to the Murphy eye at the distal end of the ETT when inserted through the valve port connector on the 
Table 1. Visual Representation of Premeasured Distances and Measured Values Used to Estimate Distance From ETT Tip to Carina

\begin{tabular}{|c|c|c|c|}
\hline ETT Size, mm & $\begin{array}{c}\text { Valve Port on Ventilator } \\
\text { Circuit to Proximal } \\
\text { Margin of ETT Cuff, cm (A) }\end{array}$ & $\begin{array}{c}\text { Visual Representation } \\
\text { of Premeasured } \\
\text { Distances }\end{array}$ & $\begin{array}{c}\text { Proximal Margin } \\
\text { of ETT Cuff to } \\
\text { Distal Tip of ETT, cm (B) }\end{array}$ \\
\hline 3.0 & 18.8 & & 2.3 \\
\hline 3.5 & 20.8 & & 2.3 \\
\hline 4.0 & 22.3 & mementer & 3.3 \\
\hline 4.5 & 24.5 & A & 3.8 \\
\hline 5.0 & 25.5 & & 4.0 \\
\hline 5.5 & 27.5 & & 5.0 \\
\hline 6.0 & 29.0 & & 5.0 \\
\hline 6.5 & 29.8 & B & 5.3 \\
\hline
\end{tabular}

ventilator circuit. Prior to subject enrollment, the researchers validated these measurements by inserting each optical fiber available for use in the study through the valve port of a ventilator circuit connected to the intended ETT.

For the purposes of this study, a properly positioned ETT had the proximal border of the ETT cuff at the level of the sternal notch, defined for this study as an imaginary line connecting the superior margins of the clavicular heads. The sternal notch was chosen as the location for the acoustic detector because it is an easily identifiable surface landmark that coincides with the superior margin of the thoracic trachea and is located approximately $60 \%$ of the distance between the carina and the vocal cords. ${ }^{15}$ Approximations of tracheal length were based on published studies, which report ranges in length of $4-6 \mathrm{~cm}$ in infants and $5-10 \mathrm{~cm}$ in children up to $10 \mathrm{y}$ old. ${ }^{2}$ All ETTs used in the study were standard, uncut, cuffed, products (Mallinckrodt Medical, St. Louis, Missouri). In addition to recording the distance from the proximal margin of the ETT cuff to the ETT distal tip, we also recorded the distance from the optical fiber's insertion point at the valve port on the ventilator circuit to the proximal margin of the ETT cuff (Table 1). These measures were used offline to calculate ETT depth during the data collection.

Four respiratory therapists were instructed and successfully completed a study procedure competency using a low-fidelity training mannequin. Under the direct supervision of the study investigators, the respiratory therapists performed the procedure and data collection.

\section{Data Collection}

Subjects were placed on a chest radiograph plate with the head held midline in a neutral position so that ETT position would be determined under identical conditions for both $\mathrm{OA}$ and chest radiograph measurements. The ap- propriately sized optical fiber was selected for each subject (eg, an optical fiber labeled for a 3.0-mm ETT was used for a subject intubated with a 3.0-mm cuffed ETT). After placing a small amount of ultrasound gel on the skin, the acoustic detector was placed in the subject's sternal notch; the detector was held in place by hand during the procedure, and then it was secured in place with a small strip of NeoBond Hydrocolloid tape (Neotech, Valencia, California) during chest radiograph. The sterile $1.0-\mathrm{mm}$ diameter optical fiber was then advanced through the valve port on the ventilator circuit through the ETT. During optical fiber insertion and data capture, the subject continued to receive mechanical ventilation and oxygen administration as prescribed by the clinical team. The duration of the procedure was 30-45 s, comparable to the time used for ETT suctioning. Children were monitored during measurements per usual care with cardiorespiratory monitoring, capnography, and pulse oximetry. Changes in cardiorespiratory parameters, including vital signs, $\mathrm{S}_{\mathrm{pO}_{2}}$, and end-tidal carbon dioxide pressure, and adjustments in ventilator settings, including tidal volume, peak inspiratory pressure, mandatory frequency, and $\mathrm{F}_{\mathrm{IO}_{2}}$ during and immediately following OA measurement were noted.

After the optical fiber was advanced to the distal rim of the ETT cuff, it was withdrawn until peak graphic signal amplitude was obtained, indicating that the optical fiber was directly beneath the detector (Fig. 1). The distance from the valve port of the ventilator circuit to the maximum acoustic signal was calculated using the premeasured distance from the valve port on the ventilator circuit to the proximal margin of the ETT cuff displayed in Table 1. At the end of each procedure, the optical fiber was sterilized by soaking it in Cavicide (Meterx Research, Parker, Colorado) for $20 \mathrm{~min}$, rinsing with sterile water, and drying with sterile gauze. 


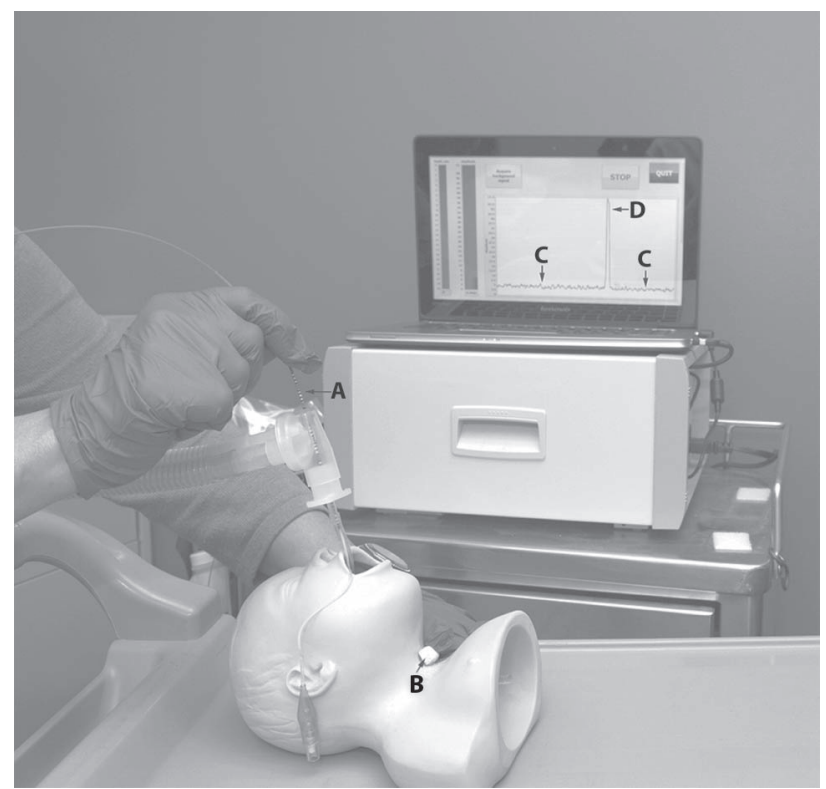

Fig. 1. A mannequin illustrates the position of $(A)$ the optical fiber within the endotracheal tube and $(B)$ the acoustic detector on the external surface of the neck at the level of the sternal notch. Serial optoacoustic signal outputs from a study patient are displayed (C) at baseline, when the optoacoustic signal is positioned proximal or distal to the acoustic detector, and (D) at maximal optoacoustic signal, when the optoacoustic signal output is immediately beneath the acoustic detector, seated at the sternal notch. The signal demonstrates a high signal-to-noise ratio.

When each chest radiograph image was displayed, the investigator drew a pair of lines on the image. The digital system provided a distance measurement for each line the investigator traced, and this was used to calculate and record the predicted distance of the ETT tip to the carina. First, the distance as predicted by OA measurement from the tip of the ETT to the acoustic detector was marked on the digital image (Measurement 1, Fig. 2). Next, the distance from the acoustic detector to the carina was measured and marked on the digital image (Measurement 2, Fig. 2). To determine the OA distance from the tip of the ETT to the carina (Calculation 1, Fig. 2), the OA distance from the tip of the ETT to the acoustic detector (Measurement 1, Fig. 2) was subtracted from the radiographic distance from the distance from the sternal notch to the carina (Measurement 2, Fig. 2).

Separately, the pediatric radiologist reported pertinent clinical findings and the position of the ETT as the distance from the ETT tip to the carina in the electronic health record. That distance was recorded for comparison to data obtained from the OA calculation.

\section{Statistical Analysis}

Data were analyzed using SAS 9.4 (SAS Institute, Cary, North Carolina). Descriptive statistics reported population

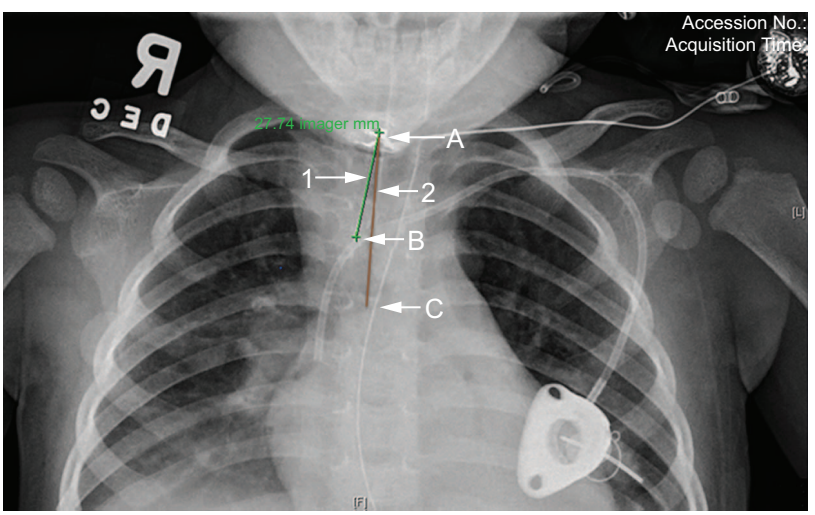

Fig. 2. Chest radiograph illustrating the measurements and calculations from the study. (A) Sternal notch; (B) tip of the ETT; (C) carina. The numbers represent the measurements made after the optical fiber was withdrawn from the ETT: (1) Calculated distance from A to B with optoacoustics; (2) measured distance from A to $C$ with chest radiograph; (3) measured distance from $B$ to $C$ with chest radiograph. The distance from ETT tip to carina by optoacoustics was determined by subtracting measurement 1 from measurement 2. ETT = endotracheal tube.

demographics. The Pearson correlation coefficient was used to assess the relationship between the distances from the tip of the ETT to the sternal notch as determined by the pediatric radiologist's review of the chest radiograph and OA techniques. Paired $t$ tests evaluated potential differences in ETT distance from ETT tip to sternal notch as determined with chest radiograph and OA. A Bland-Altman plot assessed agreement between $\mathrm{OA}$ and chest radiograph distance from the ETT tip to the sternal notch. Post hoc analyses included the two 1-sided tests (TOST) method for equivalence testing with clinically relevant, highly rigorous and moderately rigorous clinically relevant epsilons along with corresponding sample size and power analyses. Statistical significance was established at $P<.05$.

\section{Results}

\section{Population Characteristics}

Twenty-six children were enrolled in the study (Table $2)$. Most of the subjects were white $(65.4 \%)$ and male $(57.7 \%)$. Subjects ranged in age from $<1$ month after a term birth to $11 \mathrm{y}$, with median (interquartile) age, weight, and height of 9.0 months (4.0-24.0), 9.6 kg (5.7-13.0), and $75.0 \mathrm{~cm}(62.0-90.0)$, respectively. The cuffed ETTs ranged from $3.0 \mathrm{~mm}$ to $5.0 \mathrm{~mm}$. OA and distances reported by the pediatric radiologic from the ETT tip to the carina are reported in Table 3.

\section{Comparison of ETT Position}

Incomplete data were available for 2 subjects. One study was terminated before obtaining data due to unrelated tran- 
Table 2. Description of Study Population

\begin{tabular}{|c|c|}
\hline Subjects & $26(100)$ \\
\hline \multicolumn{2}{|l|}{ Gender } \\
\hline Female & $11(42.3)$ \\
\hline Male & $15(57.7)$ \\
\hline \multicolumn{2}{|l|}{ Ethnicity } \\
\hline African American & $4(15.4)$ \\
\hline Asian & $4(15.4)$ \\
\hline Caucasian & $17(65.4)$ \\
\hline Hispanic & $1(3.8)$ \\
\hline \multicolumn{2}{|c|}{ Endotracheal tube size, $\mathrm{mm}$} \\
\hline 3.0 & $7(26.9)$ \\
\hline 3.5 & $10(38.5)$ \\
\hline 4.0 & $5(19.2)$ \\
\hline 4.5 & $3(11.5)$ \\
\hline 5.0 & $1(3.9)$ \\
\hline \multicolumn{2}{|l|}{ Age, months } \\
\hline$<1$ & $3(12)$ \\
\hline $1-3$ & $2(8)$ \\
\hline $4-6$ & $7(27)$ \\
\hline $7-9$ & $2(8)$ \\
\hline $10-12$ & $1(4)$ \\
\hline $13-15$ & $2(8)$ \\
\hline $16-18$ & $0(0)$ \\
\hline $19-21$ & $2(8)$ \\
\hline $22-24$ & $1(4)$ \\
\hline $25-36$ & $1(4)$ \\
\hline $37-48$ & $3(12)$ \\
\hline$\geq 49$ & $2(8)$ \\
\hline \multicolumn{2}{|c|}{ Anthropometric measurements, median (IQR) } \\
\hline Weight, $\mathrm{kg}$ & $9.6(5.7-13.0)$ \\
\hline Height, cm & $75.0(62.0-90.0)$ \\
\hline
\end{tabular}

sient physiologic instability. No signal was obtained in 1 subject, although the chest radiograph demonstrated that the tip of the ETT was positioned $4 \mathrm{~mm}$ cephalad to the sternal notch, so the absence of an OA signal correctly indicated ETT malposition.

In the remaining 24 subjects, the estimated ETT depth based on the OA signal was $<1 \mathrm{~cm}$ from the location identified by the pediatric radiologist (mean distance $0.1 \pm 0.34 \mathrm{~cm}$ ). The peak signal was detected in $<45 \mathrm{~s}$ for each subject. The OA measurement was associated with no adverse hemodynamic effects, such as changes in heart rate, blood pressure, end-tidal $\mathrm{CO}_{2}$, or $\mathrm{S}_{\mathrm{pO}_{2}}$. No ventilator alarms were triggered to denote significant changes in peak airway pressure or reductions in tidal volume during the procedure. There were no adjustments to ventilator settings, including mandatory frequency, $\mathrm{F}_{\mathrm{IO}_{2}}$, tidal volume, or peak inspiratory pressure required during or immediately after the study procedure.
Table 3. Distance From the ETT Tip to the Carina

\begin{tabular}{|c|c|c|c|}
\hline ETT Size, mm & $\begin{array}{l}\text { Optoacoustic } \\
\text { Distance, } \mathrm{cm}\end{array}$ & $\begin{array}{l}\text { Radiographic } \\
\text { Distance, cm }\end{array}$ & Difference, cm \\
\hline 3.0 & 1.00 & 0.5 & 0.50 \\
\hline 3.0 & 1.80 & 1.7 & 0.10 \\
\hline 3.0 & 1.90 & 1.7 & 0.20 \\
\hline 3.0 & 2.50 & 2.6 & -0.10 \\
\hline 3.0 & 1.30 & 1.0 & 0.30 \\
\hline 3.0 & 1.00 & 1.0 & 0.00 \\
\hline 3.5 & 1.40 & 1.5 & -0.10 \\
\hline 3.5 & 1.15 & 1.0 & 0.15 \\
\hline 3.5 & 3.10 & 3.8 & -0.70 \\
\hline 3.5 & 2.25 & 2.3 & -0.05 \\
\hline 3.5 & 0.70 & 0.5 & 0.20 \\
\hline 3.5 & 1.50 & 1.2 & 0.30 \\
\hline 3.5 & 1.80 & 2.0 & -0.20 \\
\hline 3.5 & 3.00 & 2.8 & 0.20 \\
\hline 3.5 & 3.00 & 3.0 & 0.00 \\
\hline 3.5 & 3.00 & 2.5 & 0.50 \\
\hline 4.0 & 2.00 & 2.2 & -0.20 \\
\hline 4.0 & 1.30 & 1.8 & -0.50 \\
\hline 4.0 & 2.30 & 2.0 & 0.30 \\
\hline 4.0 & 1.30 & 1.4 & -0.10 \\
\hline 4.0 & 2.00 & 2.0 & 0.00 \\
\hline 4.5 & 2.30 & 2.0 & 0.30 \\
\hline 4.5 & 3.00 & 2.0 & 1.00 \\
\hline 5.0 & 3.00 & 3.0 & 0.00 \\
\hline
\end{tabular}

Pearson's correlation coefficient demonstrated a strong and significant relationship between OA distance and chest radiograph distance $(r=0.91, P<.001)$. Paired $t$ test results did not provide evidence of significant differences in ETT tip distance from the carina when OA determination was compared with the radiologist's interpretation of the ETT position by chest radiograph (degrees of freedom $[\mathrm{DF}]=23$, $t=1.29, P=.21)$.

A Bland-Altman plot assessed agreement, with negligible bias, or mean difference $(0.1 \mathrm{~cm})$, and narrow $95 \%$ limits of agreement $(-0.76,+0.58 \mathrm{~cm})$ (Fig. 3). No distinct pattern of asymmetry around the zero bias line was discernible, demonstrating that any bias present was not variable. Shukla's method was used to assess the equality of precision between the 2 measures; Pearson's correlation coefficients for differences of measures and means of measures were not significant $(\mathrm{r}=0.17, P=.44)$, indicating equality of precision. Examination of the effect of ETT size on the relationship between $\mathrm{OA}$ and chest radiograph distance was limited due to the pilot study's small sample size. Visualization of data by ETT size suggests there may not be an effect; however, this observation is based solely on observation and must be verified with a larger sample size. From a clinical perspective, the close correlation between $\mathrm{OA}$ and 


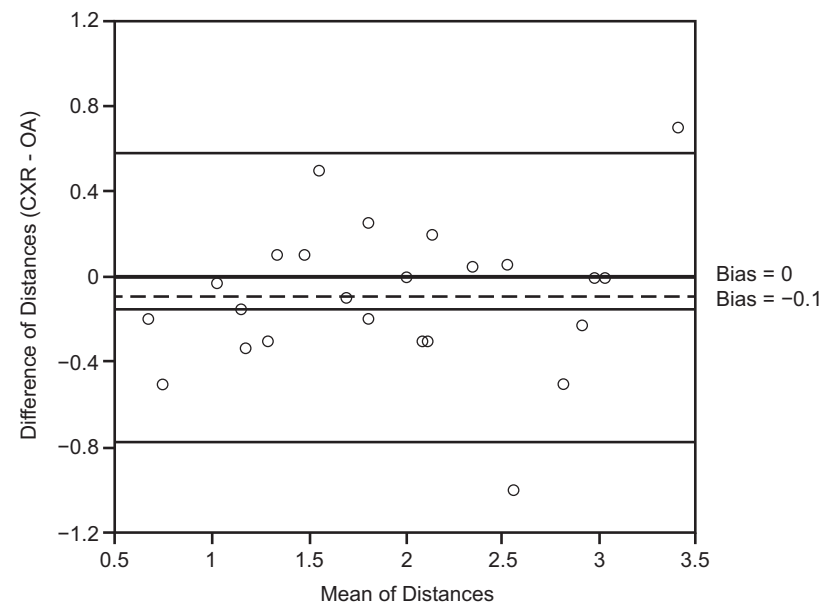

Fig. 3. Bland-Altman plot of the bias and precision of the differences between optoacoustic (OA) and chest radiographic (CXR) measurements of the distance from the sternal notch to the endotracheal tip. Points show the difference of distances. Solid horizontal lines show the 95\% upper and lower limits of agreement. Dashed line represents the bias.

chest radiograph measurements for 3.0-mm and 3.5-mm ETTs suggests that the $\mathrm{OA}$ and chest radiograph measurements are equivalent, even in the smallest subjects.

Post hoc analysis of equivalence between OA and radiograph measures was conducted with the two 1-sided tests methodology, in which epsilon $(\varepsilon)$, expressing the difference between measurements considered to demonstrate equivalence, was defined as clinically relevant $(\varepsilon=1.00 \mathrm{~cm})$, highly rigorous $(\varepsilon=\mathrm{SD} \times 0.25=0.09 \mathrm{~cm})$, or moderately rigorous $(\varepsilon=\mathrm{SD} \times 0.50=0.17 \mathrm{~cm})$. There was evidence of equivalence for the clinically relevant $\varepsilon$ (upper and lower $P$ values $<.001$ ), but not for the highly rigorous $\varepsilon$ (upper $P<.001$, lower $P=.53$ ) or the moderately rigorous $\varepsilon$ (upper $P<.01$, lower $P=.13$ ).

\section{Discussion}

Significant morbidity and mortality are associated with improperly positioned ETTs. ETTs that are too shallow may increase the propensity for unplanned extubation and the risk of laryngeal trauma, hypoxemia, and inadequate ventilation. ${ }^{16,17}$ If positioned too deeply, ETTs may cause carinal irritation, reactive bronchospasm, or endobronchial intubation, which in turn can impair gas exchange due to under-expansion of the nonventilated lung and overdistention or barotrauma of the ventilated lung. ${ }^{18}$ When at the correct depth, the distal tip of the ETT is located in the mid-tracheal region, or halfway between the superior borders of the clavicular heads and the carina, ${ }^{19}$ with the proximal end of the ETT cuff below the vocal cords. ${ }^{20} \mathrm{We}$ used the sternal notch as an easily palpable anatomic landmark that is formed by the heads of the clavicles. Prece- dents in the literature support the use of the sternal notch as the initial point of measure when determining the correct depth of an ETT by transillumination in adults ${ }^{21}$ and by ultrasound saline test in children. ${ }^{22}$

The premeasured custom optical fibers provided known lengths both from the insertion port on the ventilator circuit to the proximal rim of the ETT cuff and from the proximal rim of the ETT cuff to the distal tip of the ETT. Premeasured lengths enabled the investigators to quickly determine the position of the optical fiber in the ETT and the relationship of the proximal portion of the ETT cuff to the top of the thorax. An OA signal was not obtained in 1 subject whose ETT was above the clavicles. The study protocol did not permit movement in the acoustic detector, so the lack of a signal implied that the tube was positioned too shallowly, but the researchers were not permitted to move the acoustic detector cephalad to confirm this.

Estimated ETT depth based on the OA signal was $<1 \mathrm{~cm}$ from the location identified by the pediatric radiologist. In most of the subjects, the distance was nearly identical (mean difference $0.1 \pm 0.3 \mathrm{~cm}$ ). Potential confounding influences on identification of ETT depth by OA include misalignment between the acoustic detector and the chest radiograph plate and subject movement; however, those influences appeared to be clinically unimportant in this series.

The differences between OA determination and the radiologist's interpretation of ETT position on chest radiograph were small. There was a strong and significant relationship between OA distance and chest radiograph distance $(r=0.91, P<.001)$. This correlation, combined with narrow limits of agreement and minimal bias, demonstrate the precision of the OA technique compared to the standard chest radiograph method. The lack of significant association between the difference and the means of the measure suggests that the OA and chest radiograph measures may in fact be equally precise.

Currently, the most widely accepted methods of confirming the position of the ETT after tracheal intubation are chest radiograph and visualization of the tracheal rings and carina with a fiberoptic scope. ${ }^{20}$ Specialized equipment and training are required to use fiberoptic devices, and the presence of secretions may obscure visualization of the carina. ${ }^{23}$ chest radiograph can be time consuming, and it requires staff resources to maintain the head in proper position and exposes the subject to ionizing radiation. ${ }^{24}$ The ETT position snapshot obtained with fiberoptic bronchoscopy or chest radiograph has only short-term value because of the propensity for ETTs to migrate and become malpositioned during changes in head and body position during routine ICU care. ${ }^{25}$

Ultrasound, although less commonly used, requires time to prepare the cuff (eg, inflate with saline) before and after ultrasound assessment of ETT position, and it requires 
interpretation expertise. ${ }^{26}$ Frequent ETT cuff manipulation may make this technique cumbersome for staff and prone to error, such as failure to remove saline from the ETT cuff and replace with air after ultrasound assessment. In addition, filling the cuff with a substance (eg, saline) that was not intended to be used may cause the valve in the pilot balloon or the cuff to malfunction and may necessitate the need for re-intubation. Secretions and variable neck thickness reduce the accuracy of transillumination and increase the time required to determine ETT position by transillumination with a lighted stylet. ${ }^{27,28}$ There is also an increased cost of care when serial diagnostic imaging is used to confirm ETT placement. ${ }^{29}$ If a chest radiograph demonstrates malposition and prompts repositioning, a subsequent chest radiograph may be obtained to confirm the accuracy of the repositioning.

The investigational OA device was easy to use and required minimal preparation. Our pilot data suggest that the $\mathrm{OA}$ and chest radiograph measures may be equally precise and that the OA technique provides a quick $(<45 \mathrm{~s})$ and simple alternative to determine the position of an ETT in infants and children. The optical fiber $(1.0 \mathrm{~mm}$ in diameter) was smaller in diameter than a closed suction catheter. When the fiber was inserted through the valve port in the Y-piece portion of the ventilator circuit, ventilation was maintained, as evidenced by the absence of ventilator safety alarms of adverse changes in the patient's hemodynamic or oxygenation status. Our results were similar to the accuracy and precision of an OA prototype reported for 15 intubated adult subjects, in whom the mean \pm SD difference of the difference between measurements was $0 \pm 0.85 \mathrm{~cm} .{ }^{14}$ Although the cost of this clinical prototype has not been established, the component parts are relatively inexpensive, and its use may reduce the number of chest radiographs needed for ETT confirmation after intubation and during care. The lack of hemodynamic or ventilatory morbidities associated with use of OA in our cohort suggests that the device is safe.

\section{Limitations}

The sample size for this first assessment of an investigational, non-FDA cleared OA technology to assess ETT position in children consisted of 24 subjects. We initially targeted a sample size of 25-30 subjects, which we considered to be adequate for a pilot study. We did not perform a power analysis before the pilot study because we did not have, either from our experience or from the literature, the mean \pm SD difference between the measurements that would have permitted a conventional power analysis. A power analysis is most commonly used to estimate sample size when a difference is expected, rather than when no difference is expected. Therefore, the heart of this analysis was to estimate agreement with the Bland-Altman plot. The sample size and standard deviation determined the margin of error for our estimate of agreement, both of which are small. In addition, the limits of agreement fell well within a clinically relevant margin

of $\pm 1 \mathrm{~cm}$, indicating that the sample size coupled with the precision of our estimation was sufficient for the analysis performed.

As a secondary post hoc analysis, in advance of future study, we used the two 1-sided tests methodology to determine equivalence as well as to estimate the sample size required to suggest that 2 measurements are not equivalent. This method requires estimation or calculation of epsilon $(\varepsilon)$, which is defined as the difference between measurements that can be excluded by a given sample size, i.e., $\pm \varepsilon$ provides a "zone of equivalence." The factor $\varepsilon$ can be chosen to more or less rigorously determine whether the 2 methods are equivalent (eg, a highly rigorous $\varepsilon$ would be $\mathrm{SD} \times 0.25$, whereas a moderately rigorous $\varepsilon$ would be $\mathrm{SD} \times 0.50$ ). In this series, the SD of the paired difference between measurements is $0.34 \mathrm{~cm}$. Therefore, a more rigorous $\varepsilon$ would be $0.09 \mathrm{~cm}$ and a less rigorous $\varepsilon$ would be $0.17 \mathrm{~cm}$. Alternatively, a clinically relevant $\varepsilon$ could be chosen $(\mathrm{eg}, \varepsilon=1.00 \mathrm{~cm})$, such that any differences less than this value would be considered clinically unimportant. Given the SD in this series, assuming $80 \%$ power and an alpha $=0.05$, a minimum sample size of 22 pairs would be sufficient to detect non-equivalence for $\varepsilon=0.17 \mathrm{~cm}$, and a minimum sample size of 2 pairs would be sufficient to detect non-equivalence for a clinically relevant $\varepsilon=1.0 \mathrm{~cm}$. A sample size for a strict $\varepsilon=0.09 \mathrm{~cm}$ cannot be determined due to the width of the interval (zone of equality) being less than the mean difference of the sample. Therefore, the 24 subjects in this sample are sufficient to strongly indicate that the OA measurement is equivalent to radiographic measurement, based on a clinically relevant zone of equality.

The data suggest that this technology could reduce but does not eliminate the need for a chest radiograph to assess ETT position. However, for ETTs placed emergently outside operating rooms and for patients arriving in a pediatric ICU after intubation elsewhere, it is common practice to obtain a chest radiograph to establish ETT position. Moreover, it is the practice in many pediatric ICUs to obtain chest radiographs frequently to determine whether an ETT remains in the correct position. The use of this OA device may minimize this need, which may in turn reduce the exposure of children to radiation.

Evaluation of ETT distance was only performed in the supine position, making it difficult to extrapolate our results to children who are in a prone and semi-recumbent position. Although lack of a peak signal in 1 patient raised the suspicion of a shallowly positioned ETT, the study protocol did not incorporate a process to detect the location of shallowly positioned ETTs. The scope of this study 


\section{Determining ETT Position IN CHILDREN}

did not include the possible use of OA to guide initial ETT positioning or subsequent repositioning.

\section{Conclusions}

Pilot data suggest that $\mathrm{OA}$ and chest radiograph distances appear to be interchangeable. After future studies to validate these findings and to develop equations to automatically calculate the distance from the ETT tip to the carina, OA ETT position assessment may reduce or eliminate the need for chest radiograph for ETT position confirmation.

\section{REFERENCES}

1. Practice guidelines for management of the difficult airway. Anesthesiology 2003;98(5):1269-1277.

2. Griscom NT, Wohl ME. Dimensions of the growing trachea related to age and gender. AJR Am J Roentgenol 1986;146(2):233-237.

3. Ho AM, Aun CS, Karakar MK, The margin of safety associated with the use of cuffed pediatric tracheal tubes. Anaesthesia 2002;57(2): 173-175.

3. International Liaison Committee on Resuscitation. The International Liaison Committee on Resuscitation (ILCOR) consensus on science with treatment recommendations for pediatric and neonatal patients: pediatric basic and advanced life support. Pediatrics 2006;117(5): e955-e977.

4. Warren J, Fromm RE, Orr RA, Rotello LC, Horst HM, American College of Critical Care Medicine. Guidelines for the inter- and intrahospital transport of critically ill patients. Crit Care Med 2004; 32(1):256-262.

5. AARC Clinical Practice Guideline. Management of airway emergencies. Respir Care 1995;40(7):749-760.

6. DeBoer S, Seaver M, Arndt K. Verification of endotracheal tube placement: a comparison of confirmation techniques and devices. J Emerg Nurs 2003;29(5):444-450.

7. Friend J, Lindsey-Temple S, Gollow I, Whan E, Gera P. Review of the radiation exposure during screening of surgically implanted central venous access devices. J Pediatr Surg 2015;50(7):1214-1219.

8. Sodickson A, Baeyens PF, Andriole KP, Prevedello LM, Nawfel RD, Hanson R, Khorasani R. Recurrent CT, cumulative radiation exposure, and associated radiation-induced cancer risks from $\mathrm{CT}$ of adults. Radiology 2009;251(1):175-184.

9. Ahmed BA, Connolly BL, Shroff P, Chong AL, Gordon C, Grant R, Greenberg ML, Thomas KE. Cumulative effective doses from radiologic procedures for pediatric oncology patients. Pediatrics 2010; 126(4):e851-e858.

10. Menon K, Dundon B, Twolan BL, AlShammari S. Approach to unplanned extubations in a pediatric intensive care unit. Can J Crit Care Nurs 2015;26(3):25-29.

11. Kanthimathinathan HK, Durward A, Nyman A, Murdoch IA, Tibby SM. Unplanned extubation in a paediatric intensive care unit: prospective cohort study. Intensive Care Med 2015;41(7):1299-1306.

12. O'Connor R, Levine B. Airway management in the trauma setting. In: P Ferrera, S Colucciello, J Marx, V Verdile, and M Gibbs, edi- tors. Trauma management: an emergency medicine approach. St. Louis: Mosby; 2001:39-51.

13. Rios N, Ford P, Marques NR, Petrov I, Petrov Y, Prough D, et al. Optoacoustic assessment of endotracheal tube positioning. Crit Care Med 2014;42(12): A1530.

14. Wong DT, Weng H, Lam E, Song HB, Liu J. Lengthening of the trachea during neck extension: which part of the trachea is stretched? Anesth Analg 2008;107(3):989-993.

15. Fiadjoe JE, Nishisaki A, Jagannathan N, Hunyady AI, Greenberg RS, Reynolds PI, et al. Airway management complications in children with difficult tracheal intubation from the Pediatric Difficult Intubation (PeDI) registry: a prospective cohort analysis. Lancet Respir Med 2016;4(1):37-48.

16. Nishisaki A, Turner DA, Brown CA 3rd, Walls RM, Nadkarni VM; National Emergency Airway Registry for Children (NEAR4KIDS); Pediatric Acute Lung Injury and Sepsis Investigators (PALISI) Network. A national emergency airway registry for children: landscape of tracheal intubation in 15 PICUs. Crit Care Med 20138;41(3):874885 .

17. Crulli B, Loron G, Nishisaki A, Harrington K, Essouri S, Emeriaud G. Safety of paediatric tracheal intubation after non-invasive ventilation failure. Pediatr Pulmonol 2016;51(2):165-172.

18. Lange M, Jonat S, Nikischin W. Detection and correction of endotracheal-tube position in premature neonates. Pediatr Pulmonol 2002; 34(6):455-461.

19. Salem MR. Verification of endotracheal tube position. Anesthesiol Clin North America 2001 Dec;19(4):813-839.

20. Locker GJ, Staudinger T, Knapp S, Burgmann H, Laczika KF, Zimmerl M, et al. Assessment of the proper depth of endotracheal tube placement with the Trachlight. J Clin Anesth 1998;10(5):389-393.

21. Tessaro MO, Salant EP, Arroyo AC, Haines LE, Dickman E. Tracheal rapid ultrasound saline test (T.R.U.S.T.) for confirming correct endotracheal tube depth in children. Resuscitation 2015;89:8-12.

22. Reyes G, Ramilo J, Horowitz I, Freter AE, Husayni T, Sulayman R, Jaimovich DG. Use of an optical fiber scope to confirm endotracheal tube placement in pediatric patients. Crit Care Med 2001;29(1):175177.

23. Hauser GJ, Pollack MM, Sivit CJ, Taylor GA, Bulas DI, Guion CJ. Routine chest radiographs in pediatric intensive care: a prospective study. Pediatrics 1989;83(4):465-470.

24. Levy FH, Bratton SL, Jardine DS. Routine chest radiographs following repositioning of endotracheal tubes are necessary to assess correct position in pediatric patients. Chest 1994;106(5):1508-1510.

25. Galicinao J, Bush AJ, Godambe SA. Use of bedside ultrasonography for endo-tracheal tube placement in pediatric patients: a feasibility study. Pediatrics 2007;120(6):1297-1303.

26. Siddiqui N, Katznelson R, Friedman Z. Heart rate/blood pressure response and airway morbidity following tracheal intubation with direct laryngoscopy, GlideScope and Trachlight: a randomized control trial. Eur J Anaesthesiol 2009;26(9):740-745.

27. Masso E, Sabati S, Hinojosa M, Vila P, Canet J, Langeron O. Lightwand tracheal intubation with and without muscle relaxation. Anesthesiology 2006;104(2):249-254.

28. Price MB, Grant MJ, Welkie K. Financial impact of elimination of routine chest radiographs in a pediatric intensive care unit. Crit Care Med 1999;27(8):1588-1593.

This article is approved for Continuing Respiratory Care Education credit. For information and to obtain your CRCE

(free to AARC members) visit www.rcjournal.com

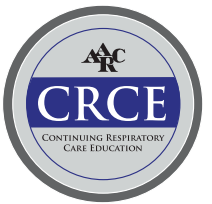

